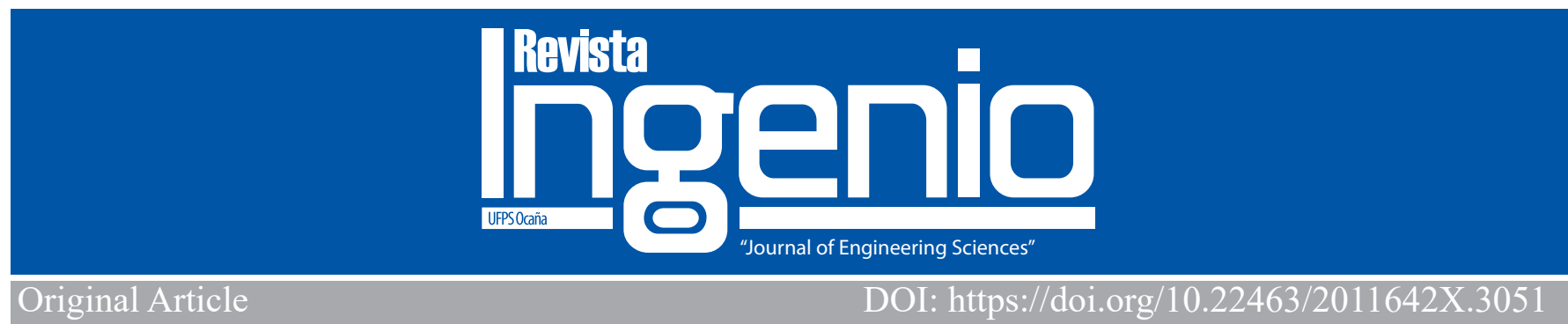

\title{
Propiedades volumétricas de mezclas asfálticas en caliente con inclusión de asfalto natural "MAPIA" y grano de caucho reciclado como llenante mineral
}

Volumetrics properties of hot mix asphalt with "MAPIA" natural asphalt and recycled rubber crumb as mineral filler

\author{
Ing. Diego Arturo Ariza-Zabala1, Ing. Carlos Daniel Sánchez-Mejía², MSc. Katerine Carreño-García ${ }^{3}$
}

${ }^{1}$ Universidad Francisco de Paula Santander Ocaña, Colombia, Orcid: https://orcid.org/0000-0002-1385-1221, Email: diegoarturoariza@gmail.com

${ }^{2}$ Universidad Francisco de Paula Santander Ocaña, Colombia, Orcid: https://orcid.org/0000-0002-6240-5711 Email: cdsanchez@ufpso.edu.co

${ }^{3}$ Grupo de Investigaciones en Transporte TRANVIA, Universidad del Norte, Colombia, Orcid: https://orcid.org/0000-0002-6553-4316, Email: kcarrenog@ufpso.edu.co

Como citar: D. A. Ariza-Zabala, C. D. Sánchez-Mejía \& K. Carreño-García, "Propiedades volumétricas de mezclas asfálticas en caliente con inclusión de asfálticas en caliente con inclusión de asfalto natural "MAPIA" y grano de caucho reciclado como llenante mineral", Revista Ingenio, vol. 19(1), pp.38-44, 2022.

Fecha de recibido: 21 de julio de 2021 Fecha aprobación:04 de novienbre de 2021

\section{RESUMEN}

Palabras claves:

La infraestructura vial es uno de los entes más importantes en el desarrollo económico de una región, ya que permite el traslado de bienes y servicios de un territorio a otro, sin embargo, este sector genera grandes afectaciones en el medio ambiente, representados en emisiones, consumo de energía y contaminación. Por tal motivo, en la actualidad se han implementado algunas alternativas

Asfalto Natural,

Estabilidad y flujo

Marshall, Grano de caucho reciclado (GCR), Llenante,

Mapia, Mezcla densa en

caliente (MDC) sostenibles que permitan la construcción/mantenimiento de vías de una manera amigable con el medio ambiente, una de ellas es la utilización de Asfalto Natural "MAPIA" para el mantenimiento de vías, sin embargo, al ser un material compuesto por poco contenido de asfalto y bastante granular, sus aplicaciones han sido sólo en vías de poco tránsito. En este orden de ideas, el presente estudio consistió en agregar "MAPIA" a una MDC-19 junto con "GCR" (Grano de caucho reciclado) con el fin de evaluar las propiedades volumétricas en base a las normativas colombianas. Se seleccionó dos porcentajes de Mapia 4 y $6 \%$ y se procedió a realizar diseño de mezclas por el método Marshall, finalmente se obtuvo valores favorables en cuanto a la estabilidad y flujo, lo que podría significar su uso en vías con altos niveles de tránsito.

\section{ABSTRACT}

Key words:

Crumb Rubber Modified (CRM), Filler, Hot Mix Asphalt (HMA), Mapia,

Natural Asphalt, Stability and Flow Marshall
Road infrastructure is one of the most important entities in the economic development of a region, since it allows the transfer of goods and services from one territory to another, however, this sector generates great effects on the environment, represented in emissions, energy consumption and pollution. For this reason, currently some sustainable alternatives have been implemented that allow the construction / maintenance of roads in an environmentally friendly way, one of them is the use of Natural Asphalt "MAPIA" for road maintenance, however as the composite material with little asphalt content and quite granular, its applications have only been in the area of low-traffic roads. In this order of ideas, the present study consisted of adding "MAPIA" to an MDC-19 together with "GCR" (Recycled rubber grain) in order to evaluate the volumetric properties based on Colombian regulations. Two percentages of Mapia 4 and $6 \%$ were selected and mixture design was carried out by the Marshall method, finally favorable values were obtained in terms of stability and flow, which could mean its favorable use in roads with high traffic.

\section{Introducción}

El desarrollo de un país está ligado a la infraestructura vial, ya que permite el transporte y movilidad tanto de productos como de pasajeros. En Colombia el $80 \%$ de la carga se moviliza por carreteras [1], sin embargo menos del $20 \%$ de la red vial nacional se encuentra en muy buen estado [2], sumado a lo anterior, estudios actuales indican que Colombia en términos de infraestructura vial se ubica en el puesto 126 entre 140 países en evaluación respecto a la calidad de sus carreteras y en los últimos lugares entre sus vecinos suramericanos [3].
Las anteriores estadísticas exteriorizan que Colombia tiene un rezago importante en términos de infraestructura vial, lo que ocasiona grandes costos logísticos, de tal forma que actualmente movilizar un contenedor desde Cartagena hacia Bogotá es tres veces más costoso que transportar uno desde el puerto de Cartagena a Shanghái en china [4], sumado a lo anterior uno de los factores más influyentes en tal déficit vial surge en el elevado costo de los materiales utilizados para la pavimentación, donde un kilómetro de carretera en américa latina puede llegar a costar hasta siete (7) veces más que el mismo tramo construido en el territorio europeo [3], entre los factores que afectan los costos se encuentra: el 
alto valor de materiales como el asfalto, por las tasas impositivas y la poca competitividad en producción, lo cual conlleva a la escasez del mismo y la necesidad de importación [6]. Adicional a lo anterior, ambientalmente, el asfalto es un material altamente contaminante, en un proceso de destilación industrial del crudo se requiere llevar el petróleo a temperaturas superiores a $\operatorname{los} 400^{\circ} \mathrm{C}$, implicando un consumo elevado de energía y emisión de gases tóxicos al ambiente, traducido en un gran impacto ambiental [7]. Esta situación pone en evidencia la necesidad de implementar nuevas tecnologías para la construcción de pavimentos flexibles, una de ellas puede ser el uso de asfaltos de origen natural como la Mapia, estos materiales son encontrados en la naturaleza en las diferentes capas terrestres y se caracterizan por ser viscosos y areniscos [8] generados a causa de fenómenos naturales de ascensión del petróleo a la superficie. Dichos materiales se pueden encontrar en estado líquido o sólido y constituyen una alternativa para construcción, mantenimiento y mejoramiento de la capas estructurales de un pavimento [9].

Estudios realizados con asfaltos naturales muestran las características y cualidades de los mismos, especialmente en la forma en que trasmiten las cargas en las distintas capas actuantes [10], presentando semejanzas significativas a las mezclas convencionales es decir con asfalto industrial [11]. Otros estudios han demostrado el aporte en las propiedades mecánicas donde se comprobó una mayor rigidez [12] y una mejor respuesta elástica [13], como también el mejoramiento en la capacidad de resistencia a los daños por humedad y el incremento en el trabajo de adhesión de le mezcla [14] además al envejecimiento [15], sin mencionar los beneficios económicos, ya que al requerir un proceso de producción más limpio, rápido y sin tanta maquinaria, son más económicos que los pavimentos convencionales[16].

En la actualidad, este tipo de mezclas se usan principalmente en vías con poco tránsito, dado sus características, escaso asfalto y gran contenido de material granular, por tal motivo, en el presente estudio, se intentó mejorar la forma en que estos pavimentos responden a las cargas, adicionando GCR (Grano de caucho reciclado) con el fin de generar un incremento en la respuesta a las cargas dinámicas y su resistencia [17], además una mayor viscosidad en proporción a la cantidad de granulo utilizado en el conglomerado asfaltico [18] y una disminución significativa en la profundidad de huella por la aplicación de cargas cíclicas que permita reducir niveles de ahuellamiento [19].

\section{Materiales y métodos}

El presente estudio tiene como objetivo el estudio y diseño de una mezcla MDC-19 compuesta por MAPIA y GCR con el fin de conocer sus propiedades volumétricas, en base a las normatividades colombianas.

\subsection{Agregados}

Los agregados pétreos provienen del municipio de la playa, Norte de Santander, producto de la trituración mecánica, el triturado pasa $3 / 4$ " con caras fracturas y bajo los estándares para el diseño de una mezcla densa en caliente MDC-19, los componentes finos y grueso fueron mezclados en base al artículo 450 de especificaciones granulométricas de agregados para mezclas asfálticas en caliente con gradación continua (INVIAS) y de acuerdo con la fórmula de trabajo, así como el porcentaje especificado conforme al diseño y ajustado a las curvas de gradación para cada grupo de muestras y combinaciones establecidas (Tabla 1), además, se realizó la caracterización de los mismos mediante pruebas físicas como gravedad específica, pesos unitarios, resistencias a degradación, índices de aplanamiento y fractura, equivalencias de arena entre otras (Tabla 2)

Tabla 1. Gradación de los agregados pétreos

\begin{tabular}{llll}
\hline $\begin{array}{l}\text { Abertura } \\
\text { Tamiz }\end{array}$ & $\begin{array}{l}\text { Triturado } \\
\text { pasa 3/4" }\end{array}$ & $\begin{array}{l}\text { Arena de } \\
\text { trituración }\end{array}$ & Control \\
\hline $3 / 4 "$ & 100,00 & 100,00 & 100,00 \\
$1 / 2 "$ & 10,85 & 100,00 & 82,17 \\
$3 / 8^{\prime \prime}$ & 1,05 & 94,97 & 76,19 \\
No,4 & 0,43 & 72,10 & 57,77 \\
No.10 & 0,36 & 52,94 & 42,42 \\
No,40 & 0,26 & 22,77 & 18,27 \\
No,80 & 0,19 & 12,96 & 10,41 \\
No,200 & 0,06 & 5,67 & 4,55 \\
\hline
\end{tabular}

Tabla 2. Propiedades físicas de agregados

\begin{tabular}{llll}
\hline Propiedades (Unid) & Norma & \multicolumn{2}{c}{ Resultado } \\
\cline { 3 - 4 } & & $\begin{array}{l}\text { Ag. } \\
\text { Fino }\end{array}$ & $\begin{array}{l}\text { AG. } \\
\text { Grueso }\end{array}$ \\
\hline $\begin{array}{l}\text { Gravedad } \\
\text { especifica (g/cm3) }\end{array}$ & INV.E-223- & 2,43 & 2,66 \\
$\begin{array}{l}\text { Gravedad } \\
\text { especifica Bulk } \\
\text { (g/cm3) }\end{array}$ & INV.E-225- & 1,89 & 1,59 \\
& 13 & & \\
Absorción (\%) & INV.E-223- & & \\
& INV.E-222- & 2,04 & 1,18 \\
& 13 & & \\
\hline
\end{tabular}

\subsection{Asfalto}

El asfalto utilizado para la realización de las muestras control o patrón es producido en la refinería de Barrancabermeja, Santander, con una penetración $60 / 70 \mathrm{dmm}$ y facilitado por la empresa Viascol SA.

Para el estudio del cemento asfaltico, se realizó caracterización con el fin de conocer sus propiedades y especificaciones mediante pruebas de: punto de ablandamiento, ductilidad, penetración, viscosidad cinemática y dinámica en un rango de temperaturas entre los $100-170^{\circ} \mathrm{C}$, 
posteriormente, se obtuvo temperaturas de mezclado y compactación, 150 y $140{ }^{\circ} \mathrm{C}$ respectivamente, teniendo en cuenta las normativas colombianas. (Tabla 3 )

Tabla 3. Propiedades físicas del asfalto

\begin{tabular}{|c|c|c|}
\hline Propiedades (Unid) & Norma & Resultado \\
\hline límite de Mezcla $\left({ }^{\circ} \mathrm{C}\right)$ & INV.E-717-13 & 150 \\
\hline $\begin{array}{l}\text { Límite de compactación } \\
\left({ }^{\circ} \mathrm{C}\right)\end{array}$ & INV E-717-13 & 140 \\
\hline Ductilidad $(\mathrm{Cm})$ & INV.E-702-13 & 150 \\
\hline $\begin{array}{l}\text { Punto de ablandamiento } \\
\left({ }^{\circ} \mathrm{C}\right)\end{array}$ & & \\
\hline$\left({ }^{\circ} \mathrm{C}\right)$ & INV.E-712-13 & 47.5 \\
\hline Penetración $(\mathrm{dmm})$ & INV.E-706-13 & $60-70$ \\
\hline Viscosidad Saybolt (S) & INV.E-714-14 & 4"12' \\
\hline
\end{tabular}

2.3 Grano de Caucho Reciclado (GCR)

El GCR producto se obtuvo proceso de trituración mecánica de llantas y neumáticos en desuso, en el municipio de Rio Negro, Antioquia, la adición de GCR brinda propiedades a la mezcla en factores dinámicos, resistencia, entre otros. Se realizó gradación para conocer la distribución granulométrica (Tabla 4) y mediante un análisis elemental (SEM) se determinó los elementos químicos en las muestras (Tabla 5)

Tabla 4. Gradación GCR

\begin{tabular}{ll}
\hline $\begin{array}{l}\text { Abertura } \\
\text { Tamiz }\end{array}$ & \% pasa \\
\hline No.10 & 100,00 \\
No,20 & 72,20 \\
No,30 & 62,90 \\
No, 40 & 22,60 \\
No,60 & 12,40 \\
No,100 & 9,60 \\
No,200 & 0 \\
\hline
\end{tabular}

Tabla 5. Composición química GCR

\begin{tabular}{ll}
\hline Elemento & Composición (\%) \\
\hline $\mathrm{C}$ & 86,9 \\
$\mathrm{O}$ & 6,37 \\
$\mathrm{~S}$ & 4,37 \\
$\mathrm{Zn}$ & 2.37 \\
\hline
\end{tabular}

\subsection{MAPIA}

La MAPIA se obtuvo de la mina "La milagrosa" en el municipio de Norcasia en el departamento de Caldas (Figura 1) [21]. Dicha mezcla de origen natural, está definida como un conjunto de agregados minerales con diversos tamaños y asfalto, los finos y gruesos en una proporción entre un (87-90\%) y un (13-10\%) de asfalto producto de procesos de refinación de origen natural (Tabla 6). A la muestra de áridos extraída con centrífuga se le realizó ensayo de gradación (Tabla 7), posteriormente, a través de análisis con microscopio de barrido electrónico (SEM) se estudió elementos químicos en las muestras, encontrando principalmente carbono, silicio, aluminio, entre otros (Tabla 8). El estudio de los elementos químicos permitiría tener un acercamiento a posibles reacciones con los materiales adicionados.

Tabla 6. Porcentaje de asfalto MAPIA

\begin{tabular}{llll}
\hline Extracción & 1 & 2 & 3 \\
\hline \% Asfalto & 10,3 & 9,72 & 10,88 \\
\cline { 2 - 4 } Promedio & 10,30 & & \\
\hline
\end{tabular}

Tabla 7. Gradación MAPIA

\begin{tabular}{ll}
\hline $\begin{array}{l}\text { Abertura } \\
\text { Tamiz }\end{array}$ & \% pasa \\
\hline $3 / 4 "$ & 100,00 \\
$1 / 2 "$ & 98,46 \\
$3 / 8^{\prime \prime}$ & 97,39 \\
No,4 & 95,79 \\
No.10 & 92,52 \\
No,40 & 48,23 \\
No,80 & 15,96 \\
No,200 & 3,59 \\
\hline
\end{tabular}

Posteriormente, al separar el material granular de la MAPIA, se obtuvo que el $95.79 \%$ del mismo pasa el tamiz\#4, es decir, corresponde a agregado fino, además, en promedio el 10.3\% del material sería un componente viscoso, similar al asfalto obtenido en procesos de refinación del petróleo.
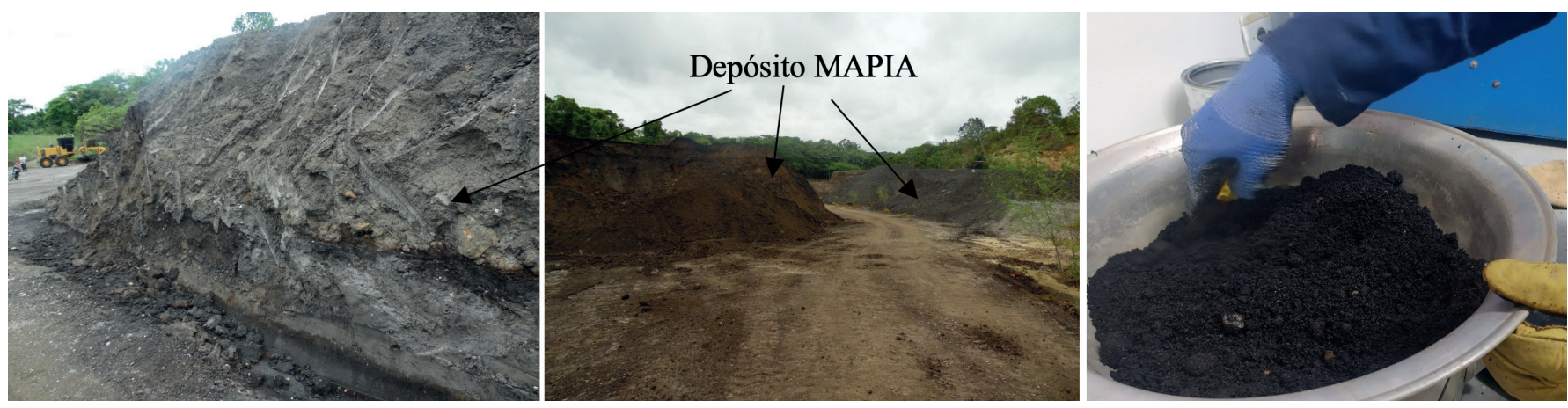

Figura 1. "MAPIA" mina la milagrosa, Norcasia. 


\section{Resultados}

El diseño experimental de esta investigación consistió en la evaluación de las propiedades volumétricas de muestras MDC-19 con MAPIA y GCR, evaluando finalmente, la resistencia del material ante la aplicación de cargas.

\subsection{Diseño Marshall}

Las mezclas se diseñaron mediante los parámetros y pautas establecidos en el método Marshall, aplicada a Colombia en la norma INV E-748-07. Se elaboraron tres especímenes para cada combinación de muestra control con cinco contenidos de asfalto en relación al peso total de la mezcla en un rango de $4-6 \%$ con intervalos de $0.5 \%$. Se desarrolló el juego de muestras compuestas por MAPIA como factor principal, agregados pétreos con granulometría seleccionada y ajustada (Tabla 8), asfalto en un porcentaje adecuado con el fin de generar y mejorar la relación de cohesión y adherencia, finalmente, se incluyó GCR como llenante mineral. Se evaluaron las propiedades volumétricas, la estabilidad y el flujo Marshall (Ver figura 2)
3.1.1 Propiedades volumétricas. Después de realizar los especímenes, se procede a determinar vacíos con aire (Va), vacíos con agregados minerales (VAM) y Vacíos con asfalto (VAF) (Tabla 9)(Ver figura 3,4 y 5).

Tabla 9. Propiedades volumétricas.

\begin{tabular}{cccccc}
\hline $\begin{array}{l}\text { \% de } \\
\text { Asfalto }\end{array}$ & $\begin{array}{c}\text { Tipo de } \\
\text { mezcla }\end{array}$ & Va & VAM & VAF & $\begin{array}{c}\text { reiaciuin } \\
\text { llenante/Asfalto } \\
\text { efectivo }\end{array}$ \\
\hline 4,0 & & 10,96 & 14,25 & 23,08 & 2,68 \\
4,5 & & 9,00 & 13,44 & 33,04 & 2,03 \\
5,0 & Control & 6,25 & 12,32 & 49,22 & 1,54 \\
5,5 & & 4,08 & 12,01 & 66,03 & 1,19 \\
6,0 & & 2,59 & 12,65 & 79,51 & 0,93 \\
\hline 4,0 & & 13,22 & 15,44 & 14,39 & 3,50 \\
4,5 & MAPIA & 10,89 & 15,30 & 28,84 & 2,71 \\
5,0 & 8,78 & 14,99 & 41,42 & 1,95 \\
5,5 & y GCR & 6,86 & 14,71 & 53,35 & 1,56 \\
6,0 & & 5,89 & 15,38 & 61,71 & 1,27 \\
\hline
\end{tabular}

Tabla 8. Adiciones de materiales.

\begin{tabular}{llllll}
\hline Material & \multicolumn{5}{l}{ Porcentajes de adición } \\
\hline Asfalto & $4,0 \%$ & $4,5 \%$ & $5,0 \%$ & $5,5 \%$ & $6,0 \%$ \\
Triturado & $25,0 \%$ & $25,0 \%$ & $25,0 \%$ & $25,0 \%$ & $25,0 \%$ \\
$3 / 4 "$ & & & & & \\
Arena de & $57,5 \%$ & $55,5 \%$ & $52,5 \%$ & $50,5 \%$ & $48,5 \%$ \\
triturado & $15,0 \%$ & $17,0 \%$ & $20,0 \%$ & $22,0 \%$ & $24,0 \%$ \\
MAPIA & $15,0 \%$ & $2,5 \%$ & $2,5 \%$ & $2,5 \%$ & $2,5 \%$ \\
GCR & $2,5 \%$ &
\end{tabular}

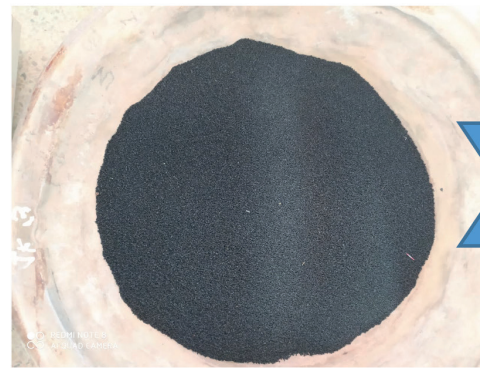

2a. Grano de caucho reciclado

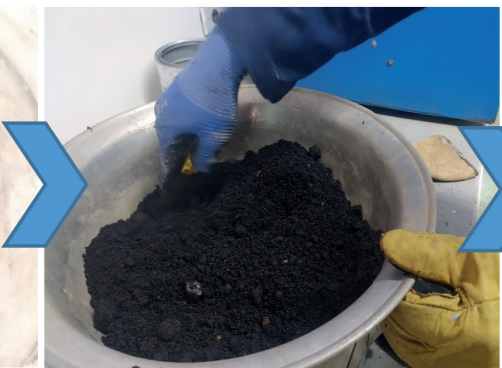

2b. MAPIA

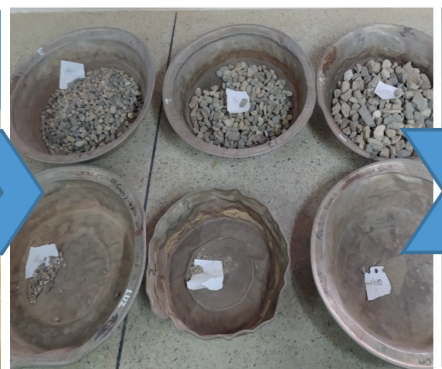

2c. Agregados naturales

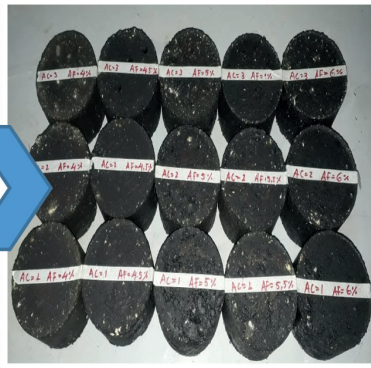

2d. Especímenes

Figura 2. Elaboración de especímenes Marshall 

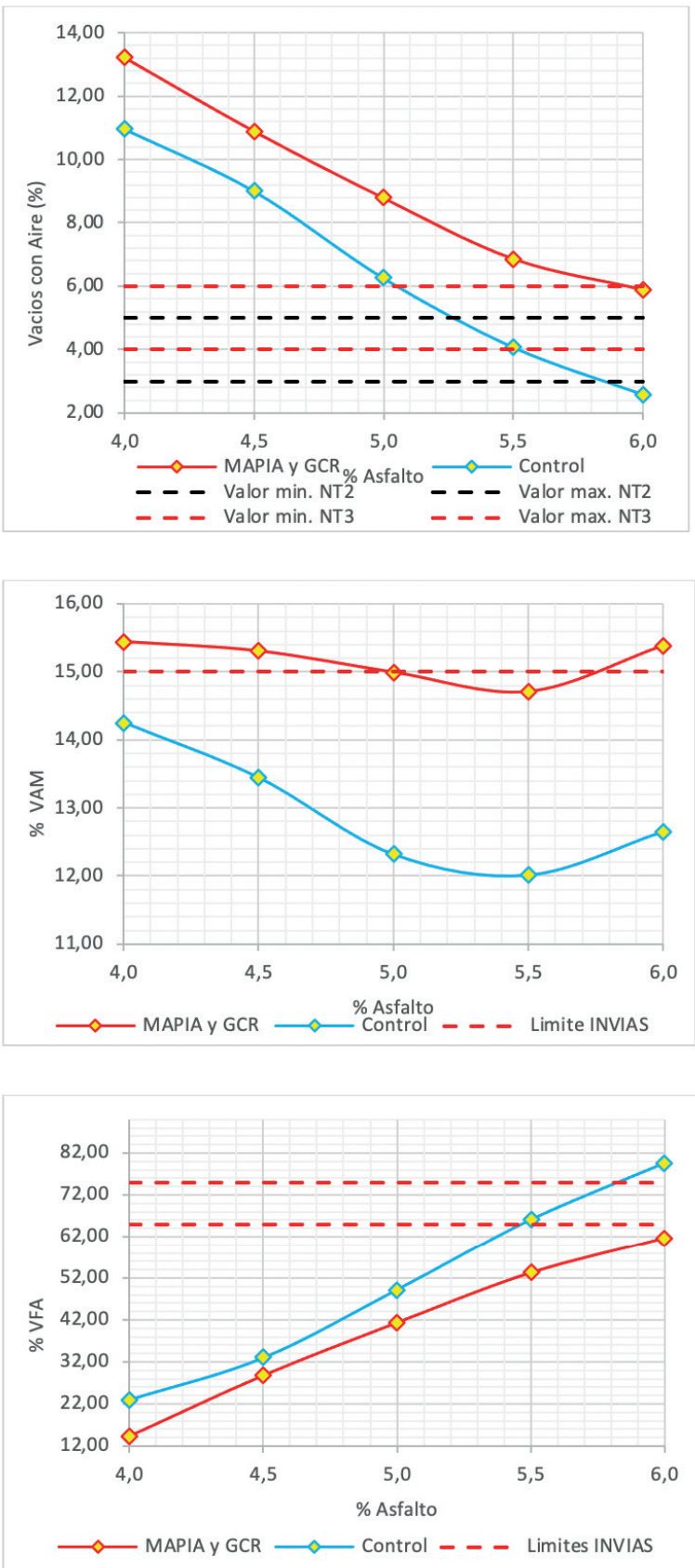

Figura 3, 4 y 5. Propiedades volumétricas mezclas asfálticas.

De las figuras 3,4 y 5 se puede deducir que las propiedades volumétricas se afectan significativamente con la inclusión de la mezcla asfáltica natural, en los vacíos con aire no cumple para un NT2 y NT3, lo mismo sucede en los vacíos con asfalto. Dichos resultados indican que las mezclas asfálticas naturales tienen un comportamiento muy inferior a una mezcla convencional.

3.1.2 Estabilidad y flujo. Después de determinar las propiedades volumétricas de los materiales, se procede a aplicar cargas para obtener valores de estabilidad y flujo Marshall (Figura 6). Los especímenes se acondicionan de acuerdo a la norma y se evalúan en base al capítulo 4 . Artículo 450 - 13. Especificaciones INVIAS 2012.

Los valores de estabilidad se pueden observar en la Figura 6, donde se muestra que todas las mezclas cumplen con los parámetros normativos de mezclas modificadas para los niveles de tránsito NT2 Y NT3, adicional, se puede evidenciar que las líneas de tendencia de ambas mezclas realizan un recorrido similar casi en paralelo, con valores mínimos para ambas en $4 \%$ y máximos en $5 \%$ de asfalto respectivamente, todos los valores de estabilidad para la mezcla con Mapia y GCR presentan valores inferiores que la mezcla de control lo que evidencia que la incorporación de Mapia y GCR disminuye un poco los valores de estabilidad aunque sigue estando bajo los limites normativos. En cuanto al flujo, los valores obtenidos permitieron conocer las deformaciones plásticas bajo acción de cargas, encontrando una tendencia a incrementar levemente y casi que a una rata de crecimiento constante con cada contenido de asfalto como se muestra en la Figura 4, la mezcla control estuvo por debajo de las mezclas modificadas, esto puede inferir una mayor deformación plástica de la mezcla modificada con Mapia y GCR debido al alto porcentaje de vacíos.

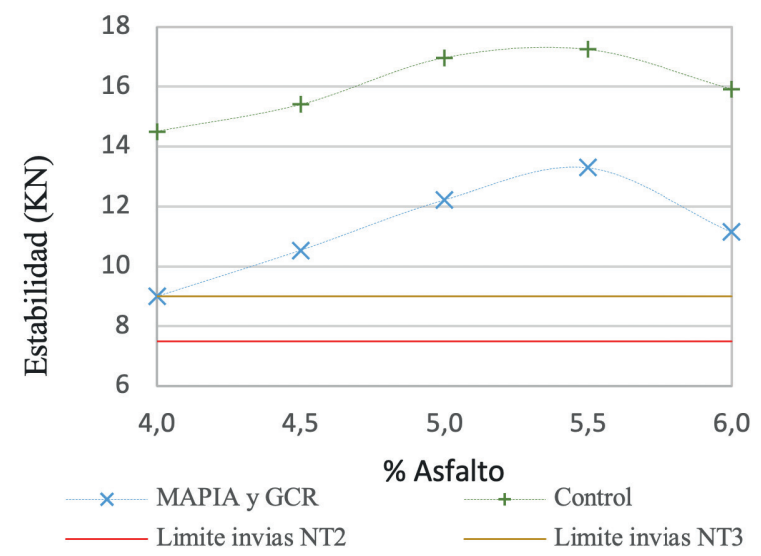

Figura 6. Valores de estabilidad en referencia a niveles de tránsito

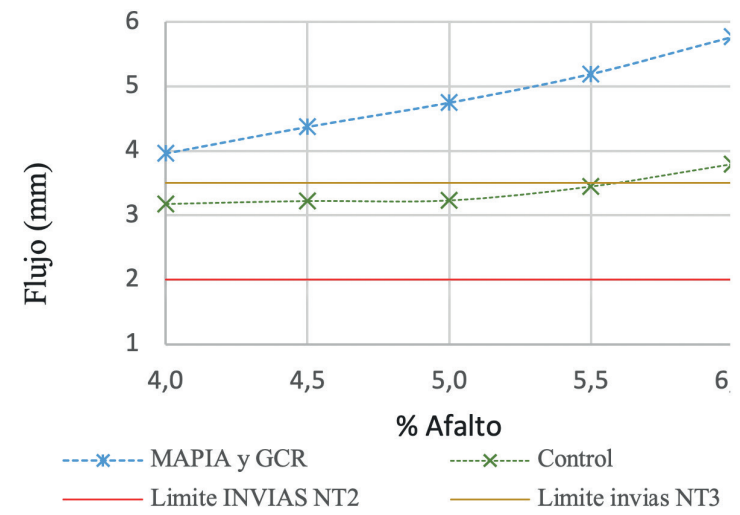

Figura 7. Valores de estabilidad en referencia a niveles de tránsito 
3.1.3 Relación estabilidad-flujo. La relación estabilidad/flujo es un parámetro que permite conocer el comportamiento del material ante la aplicación de cargas, resistencia vs deformación que experimenta, también un factor determinante de la resistencia al ahuellamiento [20].

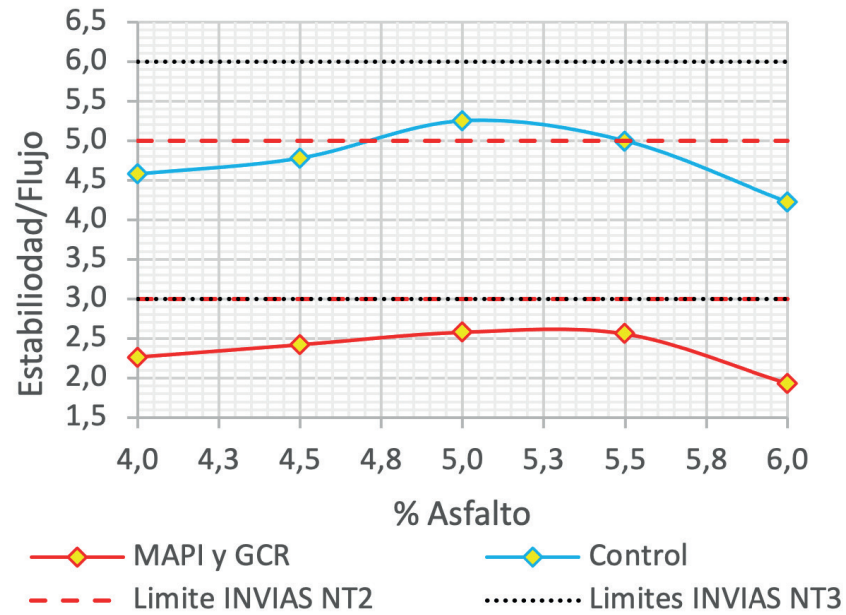

Figura 8. Relación estabilidad/flujo

En los resultados obtenidos (Ver figura 8), se encontró que la mezcla control tenía una estabilidad flujo mucho mayor que las mezclas convencionales, incluso en algunos contenidos de asfalto estaba por encima de un nivel de transito 3 (NT3), por el contrario, la mezcla modificada, no alcanzó el umbral mínimo para un tránsito NT2 ni NT3, lo que indica que, a pesar de las modificaciones, tendría un mayor potencial a deformarse por ahuellamiento que la mezcla control.

\section{Conclusiones}

El presente estudio se desarrolló con el objetivo de determinar la influencia de la inclusión de Mapia y GCR en el diseño de mezclas asfálticas en caliente tipo MDC-19, con el fin de verificar si estos materiales aportan beneficios mecánicos a los pavimentos convencionales.

A través de la aplicación del método de diseño Marshall y análisis de propiedades volumétricas, se logra deducir que la MAPIA es un material con gran potencial para la utilización en la construcción de carreteras, dado que presenta características mecánicas muy aproximadas a las requeridas por las normas en vigencia, además muestra beneficios ecológicos debido a la poca contaminación que genera su consecución y con las modificaciones adecuadas, podría cumplir con los requisitos necesarios para su aplicación en vías con niveles y solicitaciones altas de tránsito.

En cuanto a los resultados obtenidos, se encontró que las muestras con Mapia y GCR tienen mayor cantidad de vacíos con aire que una mezcla convencional, sin embargo, cumplen con los límites establecidos por INVIAS para tránsitos NT2 y NT3, los vacíos con agregado mineral cumplen satisfactoriamente para mezclas modificadas, mientras que para las convencionales no, esto puede ser explicable por el contenido de finos presente en la MAPIA. Finalmente, los vacíos con asfalto se vieron afectados directamente con la utilización del asfalto tipo Mapia, ya que no alcanzaron el umbral mínimo establecido, sin embargo, está muy cerca de ingresar dentro de las mezclas de alto módulo, dicho cambio puede significar una no satisfactoria adherencia entre las partículas, lo cual se podría estudiar con ensayos más avanzados.

En general, el GCR aporta a la mezcla beneficios ambientales reduciendo significativamente la contaminación producto de las llantas desechadas, además, provee flexibilidad a la mezcla, lo que podría indicar una mayor resistencia al ahuellamiento, pero se necesitarían estudios más avanzados para corroborar dicha hipótesis.

En cuanto a la influencia que presenta la combinación Mapia-GCR en resistencia o estabilidad, se puede concluir que cumple con los requisitos establecidos en la normatividad colombiana, aunque, los valores de estabilidad son levemente inferiores a los de la mezcla convencional. La mezcla presenta una dureza apta para su utilización con un porcentaje de 5,5\% de asfalto en total; por otra parte, se evidencia un flujo alto que puede estar relacionado con el elevado porcentaje de vacíos presentes. Cabe aclarar que la mezcla no fue modificada con ningún tipo de aditivo, por lo tanto, se espera que con el uso de ellos se pueden optimizar algunas propiedades.

Se recomienda realizar pruebas de fatiga y ahuellamiento para tener un mejor acercamiento al comportamiento de los materiales.

\section{Agradecimientos}

Agradecimientos especiales a la empresa Colombiana de Asfaltos S.A, a ViasCol S.A.S, a trituradora el Guayabal S.A, Manufactura y procesos industriales MPI.

\section{Referencias}

[1] G. J. Pérez-Valbuena, \&quot;La infraestructura del transporte vial y la movilización de carga en Colombia\&quot;, Banco de la República, Bogotá, Colombia, oct. 2005. Doi: https://doi.org/10.32468/ dtseru.64

[2] INSTITUTO NACIONAL DE VIAS, \&quot;MEZCLA ASFÁLTICA NATURAL ARTÍCULO 442P - 17\&quot;. [Online]. Available:https://www.invias.gov.co/index.php/ archivo-y-documentos/documentos-tecnicos/7032mezcla-asfaltica-natural-articulo-442p-17.

[3] M.A.A.Ariza,\&quot;ANÁLISISDELACANTIDAD Y EL ESTADO DE LAS VÍAS TERCIARIAS EN COLOMBIA Y LA OPORTUNIDAD DE LA INGENIERÍA CIVIL PARA SU CONSTRUCCIÓN 
Y MANTENIMIENTO\&quot;, p. 181, 2017.

[4] Cosoy,( 2015) \&quot;Por qué es tres veces más barato mandar un contenedor de Colombia a China que dentro mismo de Colombia\&quot;,BBC News Mundo.[Online].Available:https://www.bbc. com/mundo/noticias/2015/05/150425_colombia economia transporte problemas nc

[5] M.A.A.Ariza,\&quot;ANÁLISISDELACANTIDAD Y EL ESTADO DE LAS VÍAS TERCIARIAS EN COLOMBIA Y LA OPORTUNIDAD DE LA INGENIERÍA CIVIL PARA SU CONSTRUCCIÓN Y MANTENIMIENTO”, Tesis de pregrado,Universidad Católica de Colombia,2017.

[6] Chacón, 2014 \&quot;Asfalto o concreto | EL ESPECTADOR". [Online].Available:https:// www.elespectador.com/noticias/economia/asfaltooconcreto/.

[7] Y. Acevedo, Z. Nino, y A. Ramos,\&quot;Riesgos ambientales por emisiones atmosféricas en una refinería de petróleo\&quot;,vol. 17(3), p. 8, 2010.

[8] Y. D. S. Torres, \&quot;UTILIZACIÓN DE ASFALTO NATURAL EN LA CONSTRUCCIÓN DE PAVIMENTOS EN COLOMBIA: UNA RECOPILACIÓN BIBLIOGRÀFICA\&quot; p. 20, 2018.

[9] Colasfaltos, (2016)\&quot;Colombiana de Asfaltos S.A\&quot; [Online]. Available:http://www. colombianadeasfaltos.com/

[10] E. Santagata, P. P. Riviera, y D. D a $1 \mathrm{~m}$ a z z o , \& qu o $\mathrm{t}$; P e r formance-Relat ed Characterization of Bituminous Binders and Mixtures Containing Natural Asphalt\&quot;, Procedia - Social and Behavioral Sciences, vol. 53,pp. 535-545, oct. 2012. Doi: https:// doi.org/10.1016/j.sbspro.2012.09.904

[11] G. H. Shafabakhsh, M. Sadeghnejad, y Y. Sajed, \&quot;Case study of rutting performance of HMA modified with waste rubber powder\&quot; Case Studies in Construction Materials, vol. 1, pp. 69-76, dic. 2014. Doi: https://doi.org/10.1016/j. cscm.2014.04.005

[12] M. Yilmaz y M. E. Çeloğlu, \&quot;Effects of SBS and different natural asphalts on the properties of bituminous binders and mixtures\&quot; Construction and Building Materials, vol. 44, pp. 533-540, jul.2013. Doi: https://doi.org/10.1016/j. conbuildmat.2013.03.036

[13] I. Widyatmoko y R. Elliott, \&quot;Characteristics of elastomeric and plastomeric binders in contact with natural asphalts\&quot; Construction and Building Materials, vol. 22(3), pp. 239249, mar.2008. Doi:https://doi.org/10.1016/j. conbuildmat.2005.12.025

[14] F. Ma, Z. Fu, y L.-L. Wang, \&quot;AsphaltAggregate Adhesion Work of Natural Asphalt
Modified Asphalt\&quot; International Journal of Pavement Research and Technology, vol. 7(6), pp. 456-460, nov.2014. Doi:https://doi.org/10.6135/ijprt. org.tw/2014.7(6). 456

[15] L. F. A. Peña, \&quot;MONOGRAFIA PRESENTADA COMO REQUISITO PARA OPTAR EL TITULO DE: "ESPECIALISTA EN INGENIERIA DEPAVIMENTOS\&quot;; p. 27, 2014.

[16] B. Kök, M. Yilmaz, P. Turgut, y N. Kuloğlu, \&quot;Evaluation of the mechanical properties of natural asphalt-modified hot mixture\&quot; International Journal of Materials Research, vol. 103, pp. 506-512, abr. 2012. Doi: https://doi.org/10.3139/146.110654

[17] H. B. Takallou, R. G. Hicks, y D. C. Esch, \&quot;EFFECT OF MIX INGREDIENTS ON THE BEHAVIOR OF RUBBER-MODIFIED ASPHALT MIXTURES\&quot; in Transportation Research Record, vol. 1986(1096). Accedido: may 30, 2020. [Online].Available: https://trid.trb.org/view/284307

[18] S.-J. Lee, C. K. Akisetty, y S. N. Amirkhanian, \&quot; The effect of crumb rubber modifier (CRM) on the performance properties of rubberized binders in HMA pavements\&quot; Construction and Building Materials, vol. 22(7), pp. 1368-1376, jul.2008.Doi: https://doi.org/10.1016/j.conbuildmat.2007.04.010

[19] F. Xiao y S. Amirkhanian, HP-GPC \&quot;Approach to Evaluating Laboratory Prepared Long-Term Aged Rubberized Asphalt Binders", p. 48, 2009. Doi:https:// doi.org/10.1061/41043(350)6

[20] A. Modarres, M. Rahmanzadeh, y P. Ayar, \&quot;Effect of coal waste powder in hot mix asphalt compared to conventional fillers: mix mechanical properties and environmental impacts\&quot; Journal of Cleaner Production, vol. 91, pp. 262-268,mar.2015. Doi: https://doi.org/10.1016/j.jclepro.2014.11.078

[21] M. Gómez-Galván, R. Gallardo-Amaya, y A. A. Macgregor-Torrado, "Pavimentación con asfalto natural "MAPIA". Estudio de caso: Proyecto mejoramiento de la vía El Diviso - Torcoroma del municipio de San Martin, Cesar", Rev. Ingenio, vol. 16, n. $^{\circ} 1$, pp. 10-15, ene. 2019. Doi: https://doi. org/10.22463/2011642X.2334 